Ann. Biol. anim. Bioch. Biophys., 1977, 17 (4), 589-596.

\title{
Etude expérimentale qualitative de l'absorption des glucides après ingestion d'un repas à base de lactose chez le porc
}

\author{
par A. RÉRAT *, A. AUMAITRE **, P. VAISSADE *, P. VAUGELADE * \\ avec la collaboration technique de A. ROGER \\ * Laboratoire de Physiologie de la Nutrition \\ ** Station de Recherches sur l'Elevage des Porcs, \\ I. N. R. A., 78350 Jouy-en-Josos, France.
}

Summary. Qualitative carbohydrate absorption in pigs after intake of a lactose meal.

The kinetics of lactose digestion was studied by determining the evolution of portal and arterial glycemia (reducing sugars and true glucose) in pigs fed these sugars. Four animals (47-49 kg liveweight) were fitted with permanent cannulae in the portal vein and the aorta (Rérat, 1971). After a 20-hour fasting period, the animals received a test meal containing $400 \mathrm{~g}$ lactose and $150 \mathrm{~g}$ of a mixture of nitrogen, minerals and vitamins. Carbohydrate blood level was continuously measured from $15 \mathrm{~min}$ before the meal to $8 \mathrm{hrs}$ afterwards; its mean value was expressed graphically by considering one point to equal $5 \mathrm{~min}$.

Lactose intake caused a moderate rise (from 60 to $120 \mathrm{mg} / 100 \mathrm{ml}$ ) in reducing sugars ; this is a much more limiled result than that recorded after intake of a glucose or maize starch meal. Large variations in carbohydrate levels were observed, showing alternate maximum and minimum values. The first peak of glycemia, and consequently of absorption, occurred 60 to 70 min after the beginning of the meal, as in starch intake; it was observed $30 \mathrm{~min}$ after a glucose or sucrose meal. Contrary to other sugars, the standard deviation of the time recorded at maximum and minimum glycemia varied widely after the first minimum. Two successive periods after the meal were distinguished when considering the instantaneous difference between true glucose and reducing sugar levels in the portal vein and in the carotid artery. Until about $5 \mathrm{hrs}$, portal blood enrichment was definitely less marked for true glucose than for reducing sugars (including 66 p. 100 true glucose); after $5 \mathrm{hrs}$, the reducing sugars were almost exclusively composed of true glucose (81 p. 100). Thus, much less galactose than true glucose appeared in the blood just after the meal. After 5 hrs galactose was probably almost entirely isomerized into glucose in the gut wall during absorption. It should be observed that the absorptive process has not ceased after $8 \mathrm{hrs}$ since there is still a marked difference between portal and arterial titers.

After the meal, blood lactic acid rises from $8 \mathrm{mg} / 100 \mathrm{ml}$ to $15 \mathrm{mg} / 100 \mathrm{ml}$, but does no vary much afterwards. Instantaneous porto-arterial differences are always positive, thus demonstrating that one carbohydrate fraction (lactose, glucose or galactose) is transformed into lactic acid during digestion and absorption, and that this metabolile is absorbed and used by the organism. 


\section{Introduction.}

On sait depuis longtemps que chez les monogastriques, l'ingestion d'un repas est suivie d'une augmentation provisoire du taux de divers nutriments dans le sang : hyperglycémie porte et périphérique (Von Mehring, 1877), hyperacidémie porte ef systémique (Folin ef Denis, 1912). A partir de ces observations ef des travaux qui ont suivi, nous avons successivement proposé de décrire qualitativement (Aumaitre et al., 1969), puis quantitativement (Rérat, $1971 ; 1975$ ) les phénomènes de l'absorption des oses après un repas d'épreuve chez le porc éveillé, en mesurant en continu les variations pré- ef posfprandiales de la glycémie des sangs porte et systémique, de façon parallèle à ce qui a été réalisé pour les acides aminés (Pion, Fauconneau et Rérat, 1963 ; Rérat, 1973).

Dans le cas de l'ingestion d'un sucre simple (glucose) ou d'un glucide complexe composé d'un seul ose monomère (amidon), il suffit de déterminer un composé unique dans le sang afin de décrire le double phénomène de la digestion-absorption. Le problème se complique lorsque l'animal ingère un diholoside de composition hétérogène, comme le saccharose ou le lactose. En effet certains des oses composant la molécule sont absorbés à une vitesse variable par rapport au glucose : plus vite pour le galactose et plus lentement pour le fructose selon Cori (1925). De plus, certains oses sont susceptibles de se dégrader dans le tube digestif proximal (estomac ef duodénum) en acide lactique (Michel, 1961) et même de s'isomériser en glucose dans la paroi intestinale. Ainsi chez l'homme, Saudubray et al. (1973) décrivent une augmentation du taux d'acide lactique, ainsi que du taux de glucose sanguin à la suite d'une surcharge orale en galactose.

Nous avons complété nos travaux préliminaires basés sur les différences portojugulaires (Rérat ef al., 1973), par l'étude de l'évolution simultanée des taux de glucose, de sucres réducteurs totaux ef d'acide lactique dans les sangs porte et aortique du porc recevant une ration renfermant le lactose comme seule source de glucides.

\section{Matériel et méthodes.}

Les animaux, 4 porcs castrés, ont été sélectionnés dans une gamme de poids très étroite $(47 \mathrm{à} 49 \mathrm{~kg}$ ) ; Ils ont été rendus porteurs de canules permanentes de la veine porte et de l'artère aorte par voie carotidienne (Rérat, 1975). Afin de limiter au maximum les effets résiduels du repas antérieur sur la glycémie (Rérat ef al., 1974), le repas d'épreuve a été précédé par un jeûne d'une durée égale à $20 \mathrm{~h}$. Huit à douze jours après l'opération chirurgicale, les animaux reçoivent un repas expérimental constitué de $400 \mathrm{~g}$ de lactose monohydrate additionné d'un mélange azoté, minéral et vitaminique dont la composition a été indiquée précédemment (Aumaitre ef al., 1975). Les taux de glucides, glucose vrai et sucres réducteurs (exprimés par rapport au pouvoir réducteur du glucose) sont mesurés dans les 2 vaisseaux sanguins en continu depuis $15 \mathrm{mn}$ avant le repas jusqu'à $8 \mathrm{~h}$ après, à l'aide d'un autoanalyseur Technicon à 4 voies (Vaissade, Aumaitre ef Rérat, 1973). Pour un seul animal, on dispose des valeurs de l'acide lactique total mesuré par colorimétrie dans les deux vaisseaux, en alternance toutes les $10 \mathrm{mn}$ avec le dosage du glucose vrai. 
de la teneur initiale mesurée à jeun. Le taux du glucose qui représente la presque totalité des sucres réducteurs chez l'animal à jeun, présente un maximum au même moment, beaucoup plus atténué en valeur absolue.

On observe une série de maxima et de minima après le repas dans les deux vaisseaux pour les deux composants mesurés, avec une diminution du fitre moyen en fonction du temps. Les taux de sucres réducteurs et de glucose ne retrouvent pas les valeurs initiales $8 \mathrm{~h}$ après le début de l'ingestion. Les maxima et minima sont présentés en moyenne avec leur écart type ; on exprime également les temps moyens aux maxima et aux minima ainsi que leur écart type (fig. 2). L'absorption apparaît

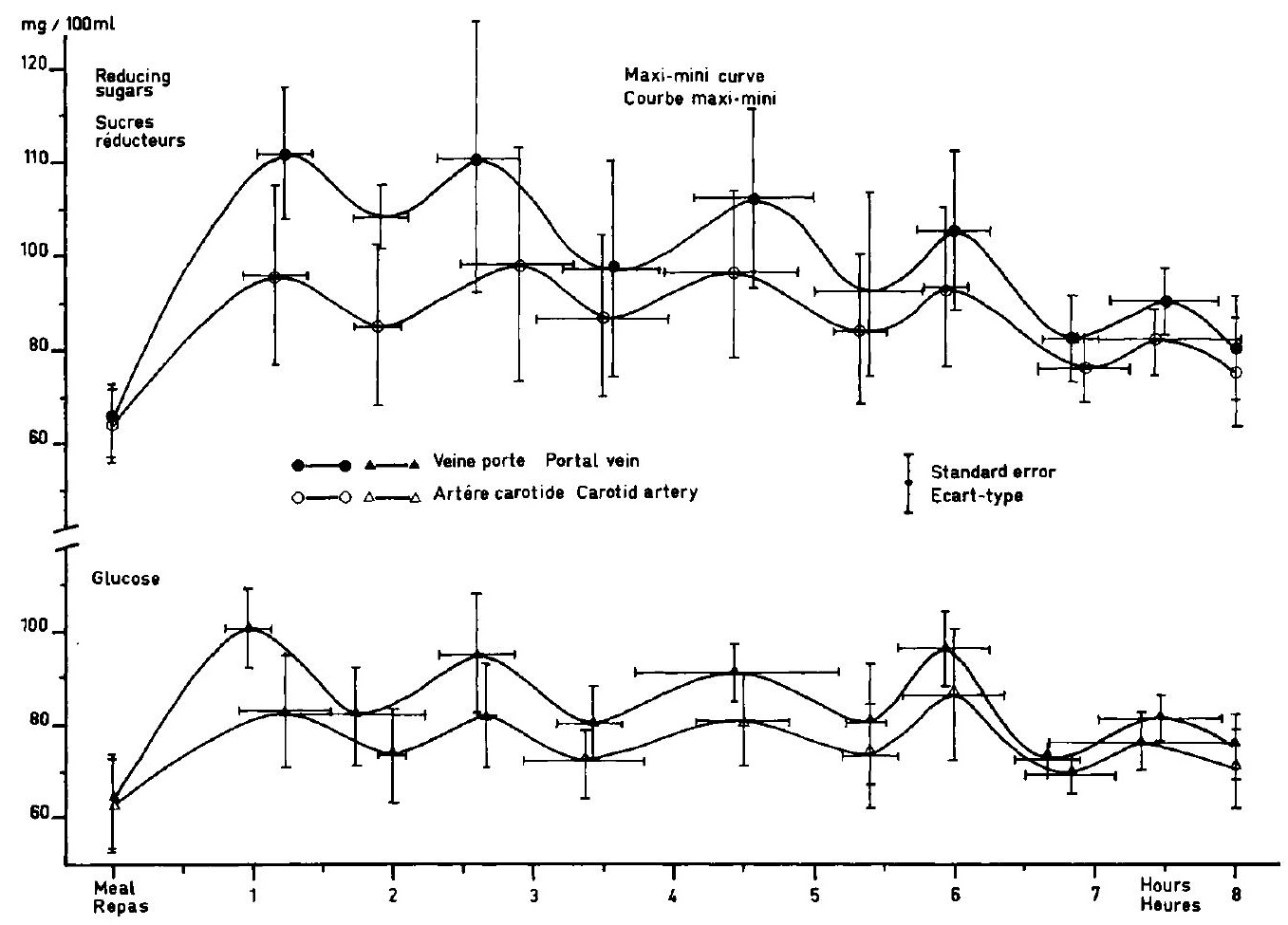

FIG. 2. - Caractéristiques et amplifude des pics d'absorption des glucides après un repas ò base de lactose : valeurs moyennes (et écarts-types) de la glycémie porte et aortique (4 animaux).

Characteristics of the curves of absorption of sugars after a lactose based diet : mean values (and standard deviation) of the portal and aortic blood levels (4 animals).

comme un phénomène discontinu car on identifie une succession de 5 maxima et 5 minima pour les deux types de glucides dans la veine porte et l'artère carotide chez les 4 animaux considérés.

Les différences porto-artérielles des concentrations pour les deux substances sont rapportées à la figure 3 . Elles sont élevées au cours des 4 premières heures 
après le repas pendant lesquelles les différences des taux de sucres réducteurs ainsi que celles du glucose sont maximales. Jusqu'à $5 \mathrm{~h}$ environ, l'enrichissement du sang porte est sensiblement moins marqué pour le glucose vrai que pour les sucres réducteurs. Après $5 \mathrm{~h}$, les substances réductrices paraissent presque entièrement composées de glucose. Les différences porto-artérielles sont toujours marquées et positives $8 \mathbf{h}$ après le début du repas.

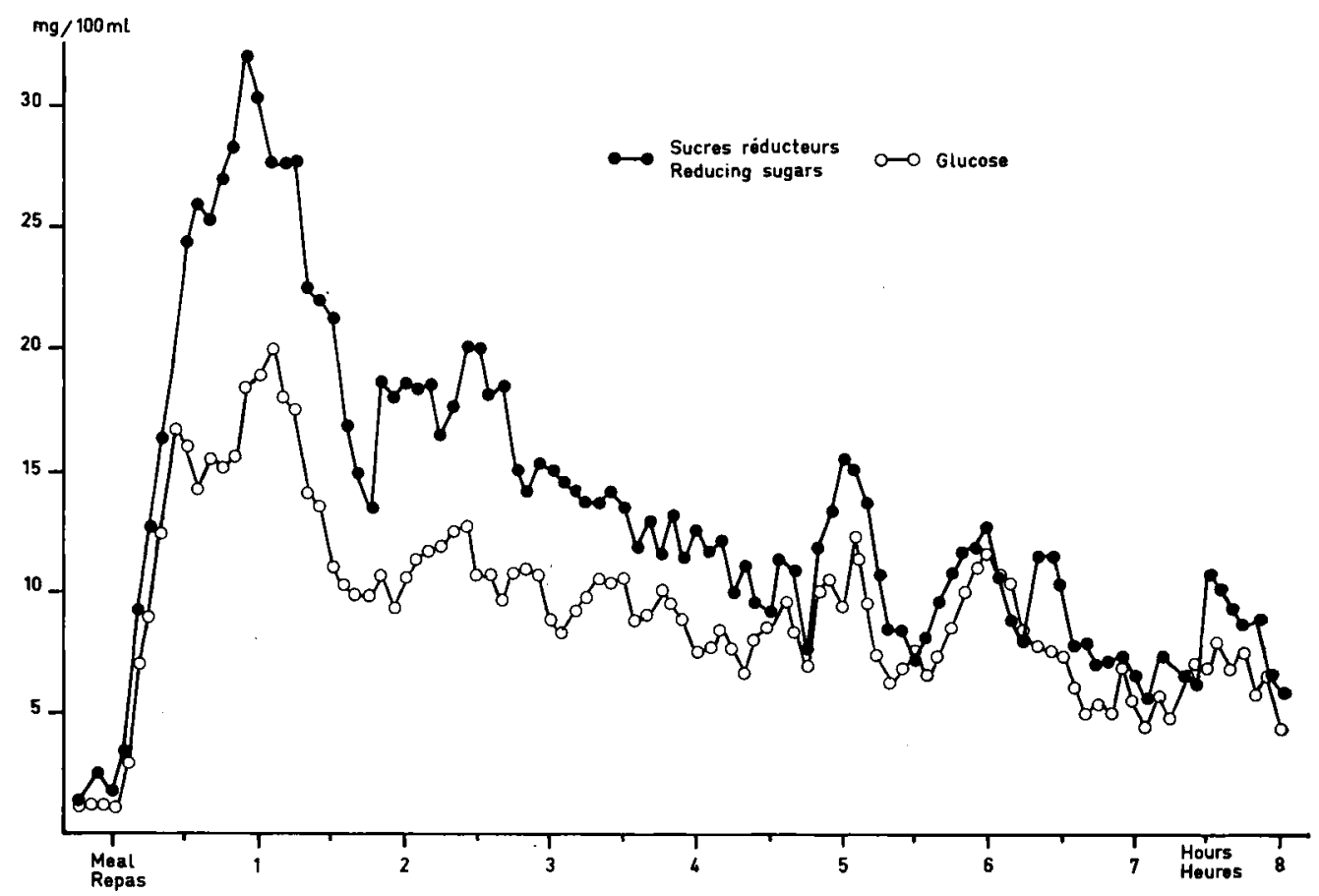

FIG. 3. - Estimation de l'évolution de l'absorption des glucides par les différences instantanées porte-aorie de concentration des sucres réducteurs ou du glucose.

Estimation of the kinetics of sugar absorption from the instant differences between portal and oortic blood concentrations of reducing sugars and glucose.

Le taux d'acide lactique mesuré sur un seul animal augmente après le repas de 8 à $15 \mathrm{mg} / 100 \mathrm{ml}$ sans toutefois présenter des valeurs successives maximales ef minimales aussi marquées que pour les autres substances.

L'expression de la proportion de glucose dans les glucides réducteurs absorbés au cours des différentes périodes postprandiales est présentée au tableau 1 : le pourcentage varie de 56 p. 100 au cours des deux h qui suivent le repas contre 77 p. 100 entre 5 ef $8 \mathrm{~h}$ après ; en moyenne il représente sur $8 \mathrm{~h}$ environ les deux tiers des sucres réducteurs. 
TABLEAU 1

Pourcentage de glucose dans les sucres réducteurs totaux à la suife d'un repas de lactose Glucose percentage in the absorbed reducing sugars (calculated from the portal-aortic differences) in the blood of the pig following a lactose based diet

\begin{tabular}{|c|c|c|c|c|c|}
\hline Temps après le repas $(h)$ & $0-2$ & $2-5$ & $5-8$ & $0-5$ & $0-8$ \\
\hline$\frac{\text { Glucose }}{\text { S. réducteurs }} \times 100 \ldots$ & 57 & 64 & 77 & 60 & 64 \\
\hline$\frac{\text { Galactose estimé }}{\text { Glucose }} \times 100$. & 77 & 56 & 30 & 66 & 56 \\
\hline
\end{tabular}

\section{Discussion et conclusions.}

L'absorption des oses issus de la digestion du lactose est un phénomène discontinu. Le résultat se retrouve dans toutes nos études en accord avec les observations déjà anciennes du Du Vigneaud et Karr (1925). II existe des variations individuelles importantes, tant en ce qui concerne la valeur du maximum de la glycémie que le temps d'apparition de ce maximum après le repas. Dans le cas du lactose, on peut attribuer cette dernière variation à des différences individuelles de l'activité lactasique intestinale, dont la déficience entraîne un allongement du délai d'apparition des nutriments glucidiques dans le sang ainsi que l'ont décrił Bond et Levitt (1976) chez l'homme.

La part du glucose dans les sucres réducteurs varie de 56 à 77 p. 100 : elle est plus élevée que la composition chimique du lactose permettrait de le prévoir. L'écart du pourcentage théorique (50 p. 100) avec le pourcentage réel observé implique que près de 30 p. 100 du galactose a été transformé en glucose (et en très faible proportion en acide lactique) au cours de la digestion-absorption, en accord avec les résultats observés chez l'homme par Saudubray et al. (1973), et conformément à nos études quantitatives précédentes (Rérat ef al., 1976). La teneur réelle du sang en galactose est plus faible que ne permet de l'estimer la différence sucres réducteurs-glucose vrai ; en effet la méthode de dosage des premiers est éfalonnée sur le glucose alors que la totalité de la différence, corrigée par la variation du pouvoir réducteur, est attribuée à la teneur du sang en galactose. Ainsi, on peut dire que la conversion du galactose en glucose est très importante entre $4 \mathrm{~h}$ ef $8 \mathrm{~h}$ après le repas, à une période où la différence porte-aorte ne dépasse pas $10 \mathrm{mg}$ de glucides pour $100 \mathrm{ml}$ de sang. Par ailleurs, on peut constater que le porc est très tolérant au galactose contrairement à l'homme ; et il reste à prouver que l'intestin ef le foie du porc sont riches en enzymes permettant la métabolisation du galactose (Métais, 1967).

L'horaire du maximum glycémique est plus tardif après le repas à base de lactose que dans le cas du glucose ou de l'amidon de maïs (Rérat et al., 1973) ef surtout dans le cas du saccharose (Aumaitre et al., 1975). Ces résultats sont en accord 
avec les hypothèses de Deuil et al. (1972) qui observent un tel phénomène chez l'homme, en rapport direct cependant avec le poids moléculaire des glucides ou leur sensibilité à l'hydrolyse digestive (maximum plus précoce pour l'amidon de riz que pour l'amidon de pomme de terre).

La durée d'absorption des produits de la digestion du lactose est relativement plus longue que celle des oses absorbés au cours d'un repas à base de glucose, d'amidon ou de saccharose, en accord avec des observations similaires chez l'homme (Bond ef Levitt, 1976). Enfin, les quantités d'acide lactique (et de lactates) absorbées au niveau du sang restent faibles, en accord avec les valeurs de Friend, Nicholson et Cunningham (1964). Selon nos résultats antérieurs (Rérat et Aumaitre, 1975 ; Rérat et al., 1976) elles seraient de $20 \mathrm{~g}$ en $8 \mathrm{~h}$ après ingestion de $400 \mathrm{~g}$ de lactose tout en restant plus faibles qu'à l'issue d'un repas à base d'amidon.

Commission CNERNA Digestion-Absorption, Tours, 13 novembre 1976.

\section{Références}

AUMAITRE A., FÉVRIER C., RÉRAT A., RIGAUD J., THIVEND P., 1969. Application de l'analyse en continu à l'étude des variations de la glycémie du sang porte au cours de la digestion chez le porc. C. R. Acad. Sc., série D, Paris, 268, 717-720.

AUMAITRE A., RÉRAT A., VAISSADE P., VAUGELADE P., 1973. Ełude expérimentale qualitative de l'absorption intestinale des glucides après ingestion d'un repas à base de glucose ef d'amidon. Ann. Biol. anim. Bioch. Biophys., 13, 784-788.

AUMAITRE A., RÉRAT A., VAISSADE P., VAUGELADE P., 1975. Mesure qualitative en continu de l'absorption intestinale des glucides : effet de l'ingestion de saccharose sur la concentration en glucose et en fructose dans le sang porte et périphérique chez le porc. Ann. Biol. anim. Bioch. Biophys., 15, 547-548.

BOND J. H., LEVITT M. D., 1976. Quantitative measurement of lactose absorption. Gastroenterology, 70, $1058-1062$.

CORI C. F., 1925. The fate of sugar in the animal body. I. The rate of absorption of hexoses and pentoses from the intestinal tract. J. biol. Chem., 66, 691-715.

DENCKER H., LUNDERQUIST A., MEEUWISSE G., NORRYD C., TRANBERG K. G., 1972. Absorption of fructose as measured by portal catheterization. Scand. J. Gastroenterology, 7, 701-706.

DEUIL R., PEQUIGNOT H., LETAILLEUR M., MUNDLER B., 1972. Le pic hyperglycémique au cours des charges glucosées ou alimentaires. Cah. Nutr. Diet., 7, 148-150.

FOLIN O., DENIS W., 1912. Protein metabolism from the standpoint of blood and tissue analysis. J. biol. Chem., 11, 87-95.

FRIEND D. W., NICHOLSON J. W. G., CUNNINGHAM H. M., 1964. Volatile fatty acid and lactic acid content of pig blood. Can. J. anim. Sci., 44, 303-308.

MEHRING (Von) J., 1877. Uber die Abzugswege des Zuckers aus der Darmhöhe. Arch. Anat. Physiol., B2, 379-415.

MÉTAIS P., 1967. Les dysglycidoses. Cah. Nuir. Diét., 2, 23-21.

MICHEL M. C., 1961. Activité métabolique de la flore bactérienne tołale isolée de l'intestin du porc. Activité des diverses espèces bactériennes. Ann. Biol. anim. Bioch. Biophys., 1, 16-28.

PION R., FAUCONNEAU G., RÉRAT A., 1963. Etude cinétique de la composition en acides aminés du sang porte chez le porc. Ann. Biol. anim. Bioch. Biophys., 3, h. s, 31-44.

RÉRAT A., 1971. Mise au point d'une méthode quantitative d'étude de l'absorption chez le porc. Ann. Biol. anim. Bioch. Biophys., 11, 277-279.

RÉRAT A., 1973. Tentative estimation of the amount of aminoacids absorbed during digestion of a protein-free diet. Proc. Nutr. Soc., 32, 50 A. 
RÉRAT A., 1975. Mesure quantitative de l'absorption des glucides après ingestion d'amidon de maïs chez le porc. Med. Chir. Diges., Sup. 2, 49-51.

RÉRAT A., AUMAITRE A., 1975. Quantitative measurement of the absorption of various polysaccharides by studying the portal-arterial differences and the portal blood flow rate in the pig. $20^{e}$ Congr. mond. Vét., Thessalonique, vol. 125 (A.S.L.).

RÉRAT A., FÉVRIER C., AUMAITRE A., VAISSADE P., VAUGELADE P., 1973. Absorption intestinale de quelques glucides chez le porc : Etude expérimentale qualitative par la mesure en continu de la glycémie porte et jugulaire. Cah. Nutr. Diét., 8, 154-156.

RÉRAT A., AUMAITRE A., VAISSADE P., VAUGELADE P., 1974. Mesure de l'absorption de glucides au cours de la digestion de l'amidon de maïs chez le porc. C. R. Acad. Sci., Paris Sér. D, 279, 831-834.

RÉRAT A., AUMAITRE A., VAISSADE P., VAUGELADE P., 1976. Absorption des produits de la digestion de quelques holosides : mesure quantitative par l'étude des différences portoartérielles et du débit de sang dans la veine porte chez le porc. 3rd Int. Congr., I. P. V. S., Ames (lowa), June 1976.

SAUDUBRAY J. M., DREYFUS J. C., CEPANEC C., LE LO'CH H., PHAM HU TRUNG, MOZZICONACCI P., 1973. Acidose lactique, hypoglycémie et hépatomégalie par déficit héréditaire en fructose-1,6-diphosphatase hépatique. Arch. Franc. Péd. 30, 609-632.

VAISSADE P., AUMAITRE A., RÉRAT A., 1973. Mesure en continu de la glycémie des sangs porte ef périphérique chez le porc éveillé : application à l'étude de l'absorption intestinale des glucides. Symp. Int. Pharm. Technicon, Paris 4-5 Octobre 1973, 18 p.

VIGNEAUD (du) V., KARR W. G., 1925. Carbohydrate utilization. I. Rate of disappearance of d-glucose from the blood. J. biol. Chem. 66, 691-715. 